Voix et Images

volxetimages

\title{
Le sarcasme dissident : une lecture politique du Libraire
}

\section{Józef Kwaterko}

Volume 7, numéro 2, hiver 1982

Michel Tremblay

URI : https://id.erudit.org/iderudit/200327ar

DOI : https://doi.org/10.7202/200327ar

Aller au sommaire du numéro

Éditeur(s)

Les Presses de l'Université du Québec

ISSN

0318-9201 (imprimé)

1705-933X (numérique)

Découvrir la revue

Citer cet article

Kwaterko, J. (1982). Le sarcasme dissident : une lecture politique du Libraire.

Voix et Images, 7(2), 385-393. https://doi.org/10.7202/200327ar d'utilisation que vous pouvez consulter en ligne.

https://apropos.erudit.org/fr/usagers/politique-dutilisation/ 


\title{
Le sarcasme dissident : une lecture politique du Libraire.
}

\author{
par Józef Kwaterko
}

C'est à la lumière de trois lectures distinctes du Libraire, faites dans des espaces-temps variés, en fonction d'un paradigme culturel et d'un cadre de lecture hétérogènes que je me suis rendu compte à quel point les notions d'«ouverture" (Umberto Eco) et de «lisibilité" (Michel Zeraffa) d'une œuvre peuvent être utiles et adéquates lorsqu'on se trouve devant le phénomène d'une écriture dont le coefficient idéologique est élevé. Je suis loin de prétendre que Le Libraire a été conçu selon un projet idéologique programmé par Gérard Bessette, il reste cependant que le microcosme social tel qu'il est cerné dans ce roman et l'existence même d'un personnage-écrivain renvoient implicitement au rapport entre l'écriture et la vie, au code socio-idéologique extralittéraire, à la contextualisation politique, etc.

"Mais commençons donc par le commencement». Il serait utile, dans un premier temps, de rappeler certaines observations faites sur le roman par la critique au Québec'. Tout d'abord, on l'a qualifié de "roman de mœurs" (Robidoux et Renaud), de "satire sociale" (Robidoux) soit de "satire métaphysique" (Glen Shortliffe). Quant à Hervé Jodoin, le personnage-narrateur, on lui attribue l'esprit "cynique et persifleur" (Robidoux), "de sarcasme" (Allard), "contagieux» (Godbout), "cabotin, acteur, humoriste, arithmomane" (Françoise lqbal). On dit que Jodoin est "le premier personnage mythique québécois" (Godbout), "l'homme de la Révolution Tranquille" (Marcotte), “un lâche... confronté à une éthique de l'enga. gement qu'il refuse" (Brochu). Ses avatars ou ascendants romanesques sont : Meursault, Roquentin, Plume, Bérénice Einberg, les personnages de Beckett, le héros-narrateur de Prochain Épisode (Jacques Allard), les protagonistes de Kafka et de Salinger (d'après les témoignages rapportés par Glen Shortliffe dans son étude).

Aucun des critiques ci-mentionnés ne se laisse prendre au traquenard de signes dilatoires récurrents dans le texte, à savoir : les "peu importe", les "aucune importance", les "comme on dit", les "ça ne veut rien dire", etc. Presque tous ont soulevé le rapport entre la mesure et la démesure dans 
le comportement de Jodoin, entre la sobriété et l'effusion verbales, entre l'emploi systématique de la litote et de l'hyperbole. Plusieurs ont situé le renversement de ce rapport (dans la structure événementielle du texte) à l'endroit de la révélation du “capharnaüm» qui, avec la vente de L'Essai sur les mœurs et la visite du curé Galarneau, constituent les événementscatalyses de l'histoire racontée. C'est en effet à partir de là que la sincérité et l'émotion commenceront à trahir la rhétorique habituelle de Jodoin et perceront son insensibilité jusqu'alors infaillible.

II est intéressant d'observer, dans la perspective où se poseront les lectures subséquentes « non québécoises » du Libraire, que certains critiques parleront - mais presque tous en termes d'échec - de l'aspect contestataire dans le comportement verbal et social de Jodoin. André Brochu établit des liens entre son engagement et l'attitude existentielle de mauvaise fo $i^{2}$. Jacques Allard parle de l'aliénation et de l'échec qui résulte de ce «combat des mots ou plus précisément de la parole (ses mots) contre la Parole (les mots)" ${ }^{3}$. Gilles Marcotte souligne la velléité et la passivité de l'action de Jodoin :

Si l'on peut concevoir une histoire de la liberté, ou des libertés, il est assez évident que Jodoin entend se tenir à l'écart de ces combats, et qu'il refuse obstinément toute forme d'engagement. II ne rêve que d'une liberté, d'une vie sans histoire(s), qu'il obtiendra en vendant frauduleusement les livres de Chicoine. (...) II est bien l'homme de la Révolution Tranquille; en lui l'idéologie du rattrapage, de la maîtrise historique ("maîtres chez nous")... est poussé jusqu'au point où elle se renverse. La vacance, l'inaction qu'organise Jodoin à la fin du roman, est l'attente d'une autre forme d'histoire ${ }^{4} . .$.

Pour sa part, Françoise lqbal renchérit en constatant l'effet « insurrectionnel w de l'usage de l'hyperbole:

Cet humour perce lorsque Jodoin entreprend de faire taire ceux qui ont droit de parole ou se l'octroient au pays de Québec, chaque contrainte au silence chez l'autre donnant lieu chez lui à un hors-d'œuvre verbal. (...) À ce point le lecteur obsenvera que chaque imposition au silence favorise une élaboration qui se prête à la caricature d'un représentant de l'idéologie prépondérante à l'époque 5 .

André Belleau en parlant du discours chez Bessette à propos de La Bagarre a noté «que le discours réaliste ne repose pas uniquement sur le caractère concret et matériel des signes mais sur le fait qu'ils sont motivés selon la vraisemblance, l'idéologie, l'histoire etc. " 6 Aussi, après cet examen trop hâtif et fragmentaire des études sur Le Libraire au Québec, serait-il utile, à des fins pratiques, de dégager quelques aspects de la réalité référentielle du texte, la situation socio-politique au Québec des années 1950, situation contemporaine de l'histoire racontée. On sait trop bien que sur le plan idéologique, le Québec, dominé par la pensée officielle, garde à ce moment son orientation conservatrice qui monopolise le réseau idéologique à l'échelle du pays écartant par là même une opposition officieuse 
ainsi que des tendances progressistes de l'heure (à la Faculté des Sciences sociales de I'Université Laval, à I'Institut canadien des Affaires publiques, autour de Cité Libre, pour n'en citer que quelques-unes). Le gouvernement Duplessis réussit à allier et à consacrer les intérêts de la bourgeoisie d'alors (les professions libérales) et de la hiérarchie catholique. La politique économique de l'Union Nationale, essentielle au développement du capitalisme - toujours aux frais d'un pillage croissant de ses ressources effectué par les industriels américains poursuit sa démarche anti-syndicaliste et, tout en valorisant le capital, exige un prolétariat docile, prêt à collaborer avec le patronat. C'est dans cette conjoncture que le clergé est devenu une des forces financières les plus importantes du pays où les institutions religieuses présentent le plus grand pouvoir d'achat'. Dans leur majorité les politiciens s'en remettent à l'Église, à une Église qui sans en avoir ni le statut ni les structures agit comme un véritable parti politique, s'étant infiltrée dans tous les domaines du temporel. Ainsi instaurée, la puissance cléricale et l'empire spirituel de son autorité s'étendent sur les domaines où le laïcat avait été particulièrement faible. On amplifie alors les censures (l'affaire Balzac), on réagit contre la menace de la liberté individuelle en prêchant le conformisme moral au nom de la doctrine chrétienne. Si on ajoute à ceci les fraudes électorales, la corruption de la loi électorale, l'achat de votes, les malversations de l'administration publique et l'intransigeance de Duplessis contre l'adoption de loi sur l'assurance-santé ("Le meilleur genre d'assurance, c'est la santé elle-même" déclarait le Chef, le 25 octobre 1955, dans Le Devoir), I'on aura un tableau suffisamment vaste de la Grande Noirceur. Puisque, lorsqu'on se penche sur Le Libraire, les problèmes de la censure et de l'emprise cléricale font les moments forts de ce roman, il serait peut-être enrichissant de rapporter deux fragments de discours que Duplessis a prononcés entre 1948 et 1950 donc à la période où Bessette situe l'histoire de son roman ${ }^{8}$ :

Je considère comme un des attributs fondammentaux d'un bon professeur le respect des lois et de l'autorité. Nous verrons à ce que les salaires et pensions ne soient payés qu'aux bons professeurs. Les professeurs n'ont pas le droit de faire la grève ni en vertu des lois humaines, ni en vertu du droit naturel.

Aucun système d'éducation ne peut être bon sans religion. Une école sans Dieu serait comme un univers sans soleil, un corps sans âme, un aigle sans ailes. Soyons donc heureux que notre enseignement soit demeuré sur une base religieuse dans notre province. L'école c'est une forteresse qui dans un monde qui nous porte à l'oublier nous rappelle la primauté du spirituel.

Assurément la personnalité de Jodoin ne correspondait pas très bien aux qualités exigées de la part d'un bon professeur au collège Saint-Étienne. Quant à l'axe métaphorique de discours de l'ancien Premier Ministre, sa courbe nous ramène sans détour aux deux lectures suivantes. 
Passons à la Tchécoslovaquie. En 1974, à Prague, les éditions Odéon publiaient Le Libraire en tchèque. Le premier tirage de huit mille exemplaires a disparu en quelques semaines. La traduction du titre en dit long sur cet attrait du public lecteur. Le titre en tchèque est "Skandal v knihkupeĉtvi» - il s'agit donc d'un scandale qui survient dans une librairie, dans une maison du livre. N'oublions pas qu'en Tchécoslovaquie, en 1974 tout autant qu'en 1980, La Chute de Camus, les écrits de Kafka, de Joyce et de Beckett, «idéologiquement hostiles", sont frappés par la censure (en Pologne, on dira de poèmes de Milosz qu'ils étaient «idéologiquement étrangers"). N'oublions pas non plus que six ans auparavant ce pays a vécu le drame que l'on sait et que le mouvement dissident n'est pas encore organisé. II faut enfin savoir que les livres des auteurs d'opposition, de Milan Kundera, de Pavel Kohut, de Vaclav Havel, taxés de révisionnisme, circulent sous forme de copies difficilement lisibles, tapees à la machine.

Eu égard à cette forme d'ostracisme qui oblitère la diffusion légale des chefs-d'œuvre de la littérature européenne et autochtone en Tchécoslovaquie, il n'est pas étonnant que la voie dans laquelle s'engage la lecture "tchèque" du Libraire soit de nature beaucoup plus spéculative qu'au Québec. L'univers de fiction romanesque étant en proche corrélation avec la réalité ambiante du quotidien, on pouvait valoriser l'action de Jodoin beaucoup plus que cela ne se faisait au Québec. Outre la sympathie que pouvait éprouver le lecteur tchèque vis-à-vis de l'aptitude de Jodoin d'ingurgiter vingt bocks de bière par soirée chez Trefflé, outre le rapprochement qu'on pouvait faire entre Jodoin et le bon soldat Chveïk de Jaroslav Haŝek (la faculté de rétorquer par des moyens totalement opposés - d'une part, un laconisme "précieux" et de l'autre une anecdote "terreà-terre» - et de rendre muet, par cette astuce, son interlocuteur, quitte à passer pour "anormal" dans les deux cas), le lecteur tchèque pouvait en effet faire siens les problèmes de la société saint-joachinoise. II pouvait y déceler une interrogation aiguë et angoissante sur son propre problème existentiel, sur son engagement ou son aliénation, enfin sur la question de la liberté intellectuelle et de l'accès à l'information?.

En Pologne, la lecture du Libraire faite par un groupe d'étudiants, par quelques amis non universitaires et ma propre lecture (en français, l'édition polonaise n'existant pas encore) a suivi celle de la Tchécoslovaquie en ce sens que nous avons constaté, nous aussi, que Jodoin fonctionnait à merveille dans notre propre contexte historique et idéologique des années 1950. II convient cependant d'ouvrir une parenthèse pour dire que le Québec des années 1950 et son premier ministre se sont identifiés en Pologne à "l'affaire des trésors" qui a bouleversé à la même époque, l'opinion publique. II s'agit des tapisseries du Château Royal de Wawel à Cracovie et de l'armure sacrée du roi Władyskaw kokietek qui pendant toute la Deuxième Guerre mondiale avaient été mises à l'abri au Québec. Lorsqu'après la Guerre le gouvernement polonais a demandé le rapatriement du trésor, Maurice Duplessis s'y est violemment opposé (il a tenu bon 
jusqu'en 1958) sous prétexte de la non-reconnaissance du régime communiste en Pologne et sous couleur de la sauvegarde de l'héritage sacré de l'Église polonaise. Au fond, il s'agissait de donner du fil à retordre à Ottawa et d'en profiter pour faire du marchandage, sur la question d'autonomie provinciale.

La spécificité de la lecture "polonaise» du Libraire - cet avertissement partiel du lecteur mis à part - était d'avoir paraphrasé à fond l'espacé-temps québécois, l'ayant transposé et resitué dans le cadre autonome des années 1950, d'avoir accordé une importance singulière aux épiphénomènes d'un malaise social général et ceci grâce aux éléments narratifs non essentiels à I'histoire racontée, enfin, d'avoir amplifié, à travers les esquisses dans le comportement de Jodoin et à travers les signes dilatoires déroutants de son journal, le contenu idéologique et la portée sociotextuelle du roman. Le trait saillant de cette lecture était l'analogie faite entre l'omniprésence cléricale et le climat conservateur d'une part, et l'omnipotence du système et de l'idéologie à l'époque stalinienne en Pologne, de l'autre. Ce mécanisme de suppléance d'une doctrine à la place d'une autre s'est produit de façon presque immédiate à travers la lecture. Un culte avait remplacé l'autre. C'est qu'en Pologne des années 1948-1956 la liberté d'un culte qui avait pour objet Staline et sa pensée était bel et bien assurée. Habitué tant bien que mal à la démagogie abrutissante qui a réduit le marxisme à une extrême austérité, imbu d'une littérature et d'une culture dithyrambistes et édifiantes à l'époque du réalisme socialiste, l'historicité faisant partie intégrante de sa conscience, le lecteur polonais sujet à l'interaction de ses propres expériences ou lectures - a pu trouver dans Le Libraire des signes manifestes ou latents suffisamment tangibles.

Si l'on veut retracer rapidement la trajectoire de cette lecture, on pourrait y discerner trois jalons essentiels (je vais parfois rapporter littéralement, telles quelles, les observations qui ont été apportées): 10

1) l'espace-temps (je me limite à trois repères)

a) Saint-Joachin est perçu comme une petite ville typique de la Pologne de la période stalinienne. La toponymie des rues (les noms des saints) ressemble à la polonaise où fourmillent les noms des Saints Martyrs du travail, de ceux qui ont excédé le plan, de ces «hommes de marbre" exploités et oubliés, genre Stakhanov et Pstrovski.

b) La librairie "Chez Léon" ressemble à celle de la province polonaise de la même période où à gauche, à la place du rayon des livres religieux et des objets de piété, se trouvent les livres édifiants, des recueils de discours et des brochures de propagande. Ici et là, ce rayon n'apporte qu'un piètre profit et les institutions bénéficient d'un important rabais (tranche du 24 mars).

c) Au bureau de placement où les chômeurs passent pour les "sanstravail», il est nécessaire d'avoir une carte attestant qu'on cherche du travail " de bonne foi" ce qui signifiait dans notre lecture qu'on devait être «idéologiquement certain et vérifié». (Cf. p. 18). 
2) les personnages

a) Hervé Jodoin a servi à la représentation d'un intellectuel déchu, hors du circuit social qui réussit à se soustraire au mensonge général érigé en système. Il décide de ne plus marcher "à coups de pied dans le cerveau» quitte à se faire condamner à une existence médiocre. Or, il rumine toujours ses idées progressistes et ses idéaux de démocratie sociale et en ce sens il peut faire figure de dissident traqué et momentanément passif. II n'attend, en fait, qu'une période d'effervescence sociale pour faire usage de ses idées "subversives" et pour s'engager ouvertement dans une cause juste. On a attribué à Jodoin certains traits de comportement et de langage propres au dissident :

- attitude de méfiance exagérée qui se traduit par le laconisme et le sarcasme. On a remarqué que Jodoin qui somnolait sur son tabouret au début finit par faire des trous dans la visière de sa casquette et par guetter le moindre mouvement dans la librairie (cf. p. 98).

- attitude de depression et de fatigue dissimulée, repérable surtout aux moments où l'acte d'écrire s'autoréfère (p. ex. «ll s'agit de tuer le temps", "ll se fait tard et j'ai le bras fatigué", "Je n'ai pas d'imagination. Je ne saurais rien inventer. Quant à ma vie passée, j'aime mieux l'oublier. ")

- attitude de jubilation étouffée qui se traduit par les tirades et le besoin de parler, de communiquer avec autrui. Ces "moments de bonheur" surviennent après la révélation du "capharnaüm", après la visite du curé et lors des rencontres avec le père Manseau. $A$ ce propos on a invoqué les passages que voici (on parlait à cette occasion de l'espoir et de la révolte) :

J'ai trouvé que, après le serrement de mains, risible si-l'on veut mais peut-être non dépourvu d'une certaine... noblesse, le patron se replongeait trop rapidement dans les détails utilitaires. J'en éprouvai de l'humeur. D'autant plus que j'étais honteux de ma tirade; honteux d'avoir perdu pied ainsi ${ }^{11}$.

Pour la première fois depuis des mois, à moins que ce ne fât des années, j'éprouvais la naïve impression que je pouvais encore servir à quelque chose; remplir un rôle utile. Ce n'était pas raisonné, évidemment. Mais j'avais beau me dire que c'était le scotch, un reflux de mon exaltation de tout à l'heure; me répéter qu'il fallait tuer cette illusion dans l'œuf, rien n'y faisait. ${ }^{12}$

Je le rassurai sur ce point en lui faisant toutefois observer que ces deux sortes de liberté (individuelle et collective) s'opposaient souvent; que, dans bien des cas, les individus dont la majorité, fatalement, exprimait l'opinion générale «officielle» étaient tellement tiraillés, ballotés par des craintes, par «certaines pressions» selon son expression, que leur prétendue liberté collective était le résultat de leurs servitudes individuelles. ${ }^{13}$

b) Léon Chicoine est identifié comme un collaborateur «éclairé » comme un intellectuel petit-bourgeois récupéré par l'appareil politique et 
qui profite des abus du dogmatisme culturel pour en tirer son avantage. Jodoin voit en lui un Homais qui "pue la mauvaise foi». Son activité à Saint-Joachin rappelle les fourberies des personnagesphilanthropes des romans positivistes polonais de la fin du $\mathrm{XIX}$ e siècle. Selon la terminologie sartrienne ce serait un "lâche", un "salaud" raffiné qui aurait pu se donner la main avec l'autodidacte de La Nausée.

c) Martin Nault passe pour un "apparatchik", un "jeune Turc" asservi à l'idéologie dominante. On souligne que Nault et Chicoine sont de vieilles connaissances dont le contact remonte à la Crise de 192933 où ils sont brassé les affaires ensemble. (cf. p. 24).

c) le père Manseau fait figure de vieux combattant communiste de l'avant-guerre, désabusé, impuissant devant la nouvelle réalité. II reste replié sur lui-même, s'adonnant à l'alcool, victime des oppressions systématiques et systématisés.

3) l'organisation du récit

a) Le journal est la forme qui répond le mieux au règlement de comptes que se propose un écrivain-dissident. Face à la menace et aux risques qu'il court le journal est une forme d'évasion où peuvent être logées en sécurité les aspirations à la liberté individuelle, collective, voire nationale.

b) le style indirect produisant l'effet de distanciation correspond parfaitement à cette forme de journal “ dissident».

c) La Libraire a fait penser à une tranche d'un grand roman picaresque moderne. Il s'agit ici d'un épisode où Jodoin, un picaro assez noble, arrive dans une petite ville avec $50 \$$, provoque un scandale en dénudant le ridicule de ses habitants pour s'en retirer, ayant empoché $1210 \$$.

II serait futile de vouloir commenter ces rapprochements plus en détail. Je voudrais cependant souligner que tous ces dénominateurs communs examinés sous l'angle d'une telle lecture - tant immédiate qu'elle fût rendent patente la question de la polysémie d'un message et de l'ouverture d'une cuvre sans pour autant témoigner d'un parti pris ou d'un projet idéologique préalable. Pour ma part, Le Libraire se rapproche de cette forme de roman réaliste du XIXe siècle que Lukács et Goldmann ont appelé Bildungsroman où la quête des valeurs authentiques est intentionnellement limitée. Cela n'empêche pas que Le Libraire soit perçu et compris comme un roman engagé. Cela pose évidemment le problème de la mimésis. On peut dire que la miméșis de chaque œuvre engagée qui exprime une position face à la réalité est déformée dans la mesure où cet engagement n'est pas traduit par une forme explicitement déclarative donc par une sorte de discours non-représentatif. Si, par exemple, la position idéologique telle qu'elle s'exprimait dans la littérature du réalisme socialiste en Pologne et dans la littérature de survivance ou du terroir au Québec était facilement définissable, c'est que dans ces œuvres la mimésis constituait un modèle qui déformait/idéalisait la réalité du vécu social qu'elles avaient pour fonction de représenter. C'était beaucoup plus un 
modèle de désirs qu'on nourrissait vis-à-vis la réalité qu'un modèle de la réalité même. En 1954, Jeanne Lapointe notait ceci dans Cité Libre :

Dans les limbes de nos romans d'idéalisation (...) s'exprime donc une volonté de survivance et de surcompensation plutôt qu'une prise de conscience. Ces œuvres nous renseignent sur nous-mêmes de façon souterraine et inconsciente, par leurs silences et leurs naïvetés plus que par leurs données explicites. ${ }^{14}$

Or, le discours du Libraire est un discours de représentation, une écriture qui met en évidence la dimension et la condition sociale de la littérature. Puisque la catégorie esthétique de mimésis repose sur l'analogie (à plusieurs degrés d'intensité allant jusqu'à l'homologie) entre l'univers fictif représenté et le monde réel, plus une œuvre est mimétique, plus sa valeur cognitive est grande. Ainsi Le Libraire pouvait avoir par le truchement de sa mimésis (supérieure, dirait Frye) une telle fonction cognitive pour ses lecteurs en Pologne et en Tchécoslovaquie. Ainsi Jodoin pouvaitil fonctionner comme un dissident déconnecté qui occasionnellement arrivait à transgresser certaines règles sociales et même briser le monolithisme idéologique. Le roman Le Libraire n'est cependant nullement une œuvre dissidente. Publié en 1960, c'est un produit du dégel post-duplessiste, c'est l'annonce de la Révolution tranquille.

1. Voici la liste des études consultées.

Allard, Jacques, “Le libraire de Gérard Bessette ou comment la parole vient au pays", Voix et images au pays, no 1, 1970, p. 51-63.

Brochu, André, "De la Bagarre aux Pédagogues", l'Instance critique, Montréal, éditions Leméac, 1974, p. 97-102.

Godbout, Jacques «Entre l'Académie et l'écurie», Liberté, vol. 16, no 93, maijuin 1974, p. 17-35.

lqbal, Françoise, «Précieux et préciosité chez Bessette : demi-mesure et démemesurem, Voix et images vol. 1, no 3, avril 1976, p. 344-347.

Marcotte, Gilles, Le Roman à l'imparfait, Montréal, éditions La Presse, 1976, p. 43-49.

Robidoux, Réjean et Renaud, André. Le Roman canadien-français du $X X_{e}$ siècle, Ottawa, Éditions de l'Université d'Ottawa, 1966, p. 104-112.

Robidoux, Réjean, "Le cycle créateur de Gérard Bessette ou le fond c'est la forme", Livres et auteurs québécois, Québec, P.U.L., 1971, p. 11-28.

Shortliffe, Glenn, "Gérard Bessette, I'homme et l'écrivain ", Études françaises, vol. 1 , no 3 , octobre 1965, p. 17-42.

2. Andre Brochu, op. cit., p. 101.

3. Jacques Allard, op. cit., p. 60.

4. Gilles Marcotte, op. cit., p. 48-49.

5. Françoise lqual, op. cit., p. 346.

6. André Belleau, Le Romancier fictif, Montréal, P.U.Q., 1980, p. 120, note au bas de la page.

7. Cf. Pierre Maheu, "Le pouvoir clérical», in Boismenu, Maillot. Rémillard, Le Québec en textes, Montréal, Boréal Express, 1980.

8. Un repere explicite dans le texte de l'édition CLF 1968, à la page 133 , situe I'histoire dans la période 1948-1950.

9. Ces observations émanent d'un groupe de jeunes traducteurs tchèques que j'ai rencontrés à Prague en 1977. 


\section{ÉTUDES 393}

10. L'édition qui a servi à la lecture était celle du CLF., poche canadien, 1968, $153 \mathrm{p}$.

11. Gérard Bessette, Le Libraire, Montréal, CLF poche, 1968, p. 48.

12. Ibid., p. 49.

13. Ibid., p. 77.

14. Jeanne Lapointe, "Quelques apports positifs de notre littérature d'imagination ", Cité libre, no 10 , octobre 1954, p. 16. 